\title{
The Numerical Range of a Continuous Mapping of a Normed Space
}

\author{
F.F. BONSAll, B.E. CAIN, and Hans SCHNeIDER ${ }^{1}$ ) (Edinburgh and Madison)
}

(Dedicated to A. Ostrowski on the occasion of his 75 th birthclay)

\section{Introduction}

Let $X$ denote a normed linear space over the real or complex field, $X^{\prime}$ the dual space of $X, G(X)$ the set of all continuous mappings of $S(X)$ into $X$, where $S(X)$ is the unit sphere of $X$, i.e. the set of all $x \in X$ such that $\|x\|=1$. Given $x \in S(X)$, let $D(x)=\left\{f \in X^{\prime}: f(x)=\|f\|=1\right\}$; and, given $T \in G(X)$, let $V(T, x)=\{f[T(x)]: f \in D(x)\}$. The numerical range $V(T)$ of a continuous mapping $T \in G(X)$ is defined by

$$
V(T)=\bigcup\{V(T, x): x \in S(X)\} .
$$

In the special case when $X$ is a Hilbert space and $D(x)$ can be identified with $\{x\}$, the numerical range of a linear operator has a long history [7]. Under the name "field of values', the concept has been extended by F. L. BAUER [1] to linear operators on all finite dimensional normed linear spaces. The numerical range of a linear operator on a semi-inner-product space has been studied by G. LUMER [4]. A normed linear space $X$ has, in general, many semi-inner-products that correspond to the norm of $X$. The choice of one of these semi-inner-products corresponds to the choice of a mapping $x \rightarrow f_{x}$ of $S(X)$ into $X^{\prime}$ such that $f_{x} \in D(x)$ for each $x$. Then the numerical range $W(T)$ for this semi-inner-product is given by

$$
W(T)=\left\{f_{x}(T x): x \in S(X)\right\} .
$$

Thus if $T$ is a continuous linear operator, $V(T)$ is the union of all the numerical ranges $W(T)$ in the sense of LUMER.

When $T$ is a continuous linear operator it is classical that $V(T)$ is a convex set if $X$ is a Hilbert space [7], but an example is given in [5] of a linear operator on a two dimensional normed linear space for which $V(T)$ is not convex. Our main result is that $V(T)$ is connected for every normed linear space $X$ and every $T \in G(X)$ (unless both $X$ is the real numbers and $T(-\alpha) \neq-T(\alpha)$ where $S(X)=\{\alpha,-\alpha\}$ ). We give two proofs of this result and include an example of a (continuous) linear operator on a real or complex two dimensional semi-inner-product space for which $W(T)$ is not connected.

1) This research was supported in part by NSF Grant GP-7073. 
Our first proof of the connectedness of $V(T)$ depends on the upper semi-continuity of the set-valued mapping $x \rightarrow D(x)$ with respect to the weak* topology on $X^{\prime}$. We show that this mapping is also upper semi-continuous for the norm topology on $X^{\prime}$ when $X=\left(c_{0}\right)$, the sup norm space of sequences converging to 0 , (and also of course when $X$ has finite dimension) but not when $X=(c)$, the sup norm space of convergent sequences, or for certain other spaces. As an alternative to upper semi-continuity we also work with a topology on the set of subsets of $X^{\prime}$, which, although it is probably less familiar than upper semi-continuity, permits us to use the class of continuous functions and their widely studied properties in place of the class of upper semicontinuous mappings. Our second proof shows that when $X$ is not the real numbers $P=\left\{(x, f) \in X \times X^{\prime}: x \in S(X), f \in D(x)\right\}$ is connected in certain topologies, and this may be of interest in itself.

\section{Connectedness of the numerical range}

Let $\mathscr{P}(U)$ denote the set of all subsets of the set $U$. If $E$ is a topological space, let $\{\mathscr{P}(U): U \subseteq E ; U$ is open $\}$ be a basis for the $\tau$-topology on $\mathscr{P}(E)$. Adjectives used with reference to the $\tau$-topology will bear the prefix ' $\tau$-', e.g. $\tau$-open. A mapping $x \rightarrow A(x)$ of a topological space $F$ into the set of subsets of a topological linear space $E$ is upper semi-continuous (usc) on $F$ if and only if for every $x \in F$ and every neighbourhood $U$ of 0 in $E$ there exists a neighbourbood $V$ of $x$ such that for all $y \in V, A(y) \subseteq A(x)+U$ (cf. [6], pages 35-36). There are other definitions of upper semi-continuity currently in use (cf. [2]). In fact, what we are calling $\tau$-continuous is sometimes called upper semi-continuous.

LEMMA 1. Let $F$ be a topological space and let $E$ be a topological linear space. If the mapping $x \rightarrow A(x)$ is $\tau$-continuous, then it is usc. If for every $x$ in $F, A(x)$ is a compact subset of $E$, then the function $A$ is $\tau$-continuous if and only if it is usc.

Proof: Assume $A$ is $\tau$-continuous. If $x \in F$ and $U$ is an open neighbourhood of 0 in $E$, then, since $\mathscr{P}(A(x)+U)$ is a $\tau$-open subset of $\mathscr{P}(E)$, it follows that $A^{-1}[\mathscr{P}(A(x)+U)]=$ $\{y \in F: A(y) \subseteq A(x)+U\}$ is an open subset of $F$. Hence $A^{-1}[\mathscr{P}(A(x)+U)]$ is a $V$ whose existence is required in the definition of usc, and $A$ is usc.

Assume that $A$ is usc. Let $\mathscr{P}(U)$, with $U$ an open subset of $E$, be a basic $\tau$-open set. If $x \in A^{-1}(\mathscr{P}(U))$ then $A(x) \subseteq U$. The compactness hypothesis now provides a neighbourhood of 0 in $E$, denoted $G$, such that $A(x)+G \subseteq U$ (see [3] pages 35 and 36 for the details). Since $A$ is usc there is a neighbourhood $V$ of $x$ such that for each $y \in V$, $A(y) \subseteq A(x)+G \subseteq U$. That is, $A(y) \in \mathscr{P}(U)$. Thus $x \in V \subseteq A^{-1}[\mathscr{P}(U)]$. So $A^{-1}[\mathscr{P}(U)]$ is open and $A$ is $\tau$-continuous.

An application of the Hahn-Banach Theorem shows that for each $x \in S(X)$ we have $D(x) \neq \emptyset$. In the weak* topology, $D(x)$ is a closed subset of the (solid) unit ball in $X^{\prime}$ and hence is compact (cf. [3] page 155). Since $D(x)$ is convex, $D(x)$ is connected 
in any topology which makes $X^{\prime}$ a topological linear space, because in any such topology $\alpha \rightarrow \alpha f+(1-\alpha) g, 0 \leqslant \alpha \leqslant 1$ is a continuous function.

LEMMA 2. Let $S(X)$ have the norm topology and let $X^{\prime}$ have the weak* topology. Then the mapping $x \rightarrow D(x)$ is $\tau$-continuous and usc.

Proof: Since $D(x)$ is compact in the weak* topology, Lemma 1 shows that it is sufficient to prove that $x \rightarrow D(x)$ is usc. Suppose that the mapping is not usc. Then there exist $x \in S(X)$ and a weak* neighbourhood $U$ of 0 in $X^{\prime}$ such that for every positive integer $n$ there exists $y_{n} \in S(X)$ and $f_{n} \in D\left(y_{n}\right)$ satisfying $\left\|y_{n}-x\right\|<1 / n$ and $f_{n} \notin D(x)+U$. Since $\left\|f_{n}\right\|=1$, there exists a weak* cluster point $g$ of $\left\{f_{n}\right\}$ with $\|g\| \leqslant 1$. Then

$$
\begin{aligned}
|g(x)-1| & \leqslant\left|g(x)-f_{n}(x)\right|+\left|f_{n}(x)-f_{n}\left(y_{n}\right)\right| \\
& \leqslant\left|g(x)-f_{n}(x)\right|+\left\|x-y_{n}\right\| .
\end{aligned}
$$

Since $\left\|x-y_{n}\right\|<1 / n$ and $g$ is a weak* cluster point of $\left\{f_{n}\right\}$, the righthand side can be made arbitrarily small by correctly choosing $n$, and so $g(x)=1$. Therefore $g \in D(x)$. However, since $g$ is a weak* cluster point of $\left\{f_{n}\right\}$ and $U$ is a weak* neighbourhood of 0 , we have $f_{n} \in g+U \subseteq D(x)+U$ for some $n$, which is contradictory.

LEMMa 3. Let $T \in G(X)$, and let the scalar field have its usual topology. Then the mapping $x \rightarrow V(T, x)$ is a $\tau$-continuous and usc mapping of $S(X)$ with the norm topology into the set of subsets of the scalar field.

Proof: Observe that $V(T, x)$, for $x \in S(x)$ and $T \in G(X)$, is compact, because it is the image of the weak* compact set $D(x)$ under the weak* continuous mapping $f \rightarrow f(T(x))$. Therefore, by Lemma 1 , it suffices to prove that $x \rightarrow V(T, x)$ is usc.

Let $x \in S(X)$ and $\varepsilon>0$, and let $U=\left\{g \in X^{\prime}:|g(T(x))|<\varepsilon / 2\right\}$. Then $U$ is a weak* neighbourhood of 0 , and so, by Lemma 2 and the continuity of $T$, we may choose $\delta>0$ such that for every $y \in S(X)$ with $\|x-y\|<\delta$ it follows that $\|T(x)-T(y)\|<\varepsilon / 2$ and $D(y) \subseteq D(x)+U$. So if $y \in S(X),\|x-y\|<\delta$, and $f \in D(y)$ then $f=g+u$ for some $g \in D(x)$ and $u \in U$. Since $g(T(x)) \in V(T, x)$ the distance from $f(T(y))$ to $V(T, x)$ is at most $|f(T(y))-g(T(x))|$ and $|f(T(y))-g(T(x))| \leqslant|f(T(y))-f(T(x))|+|u(T x)|$ $<\|T(y)-T(x)\|+\varepsilon / 2<\varepsilon$. But $f(T(y))$ was an arbitrary point of $V(T, y)$, and so $V(T, y) \subseteq V(T, x)+\{t \in$ scalar field: $|t|<\varepsilon\}$. Thus $x \rightarrow V(T, x)$ is usc.

The two proofs of the connectedness of the numerical range which we give use the connectedness of $S(X)$. Since $S(X)$ is disconnected only when $X$ is $\mathbf{R}$, the real numbers, that case is treated separately. In fact when $X=\mathbf{R}, V(T)=\{1 / \alpha T(\alpha)\} \cup$ $\{-1 / \alpha T(-\alpha)\}$ where $S(X)=\{\alpha,-\alpha\}$. This gives:

Proposition. If $X=\mathbf{R}, V(T)$ is connected if and only if $T(-\alpha)=-T(\alpha)$, where $S(X)=\{\alpha,-\alpha\}$. In particular, if $T$ is linear, $V(T)$ is connected.

Both proofs also use the following fact: If $\left\{L_{x}\right\}$ is a family of connected subsets of some topological space and if $G_{1} \cup G_{2}=\bigcup L_{x}$ is a decomposition of $\bigcup L_{x}$ into two 
non-empty disjoint sets, $G_{1}$ and $G_{2}$, open in the relative topology, then for each $x$ the entire set $L_{x}$ lies in either $G_{1}$ or $G_{2}$.

THeOREM 1. Let $T \in G(X)$. If $X \neq \mathbf{R}$ then $\mathbf{V}(T)$ is connected.

Proof: Suppose $X \neq \mathbf{R}$ and $V(T)$ is disconnected. Then $V(T) \subseteq H_{1} \cup H_{2}$, where $H_{1}$ and $H_{2}$ are open sets giving a decomposition $V(T)=G_{1} \cup G_{2}$ of $V(T)$ into disjoint, non-empty, relatively open sets $G_{i}=V(T) \cap H_{i}$ for $i=1$ and 2 . By Lemma 3 , the mapping $x \rightarrow V(T, x)$ is $\tau$-continuous, and therefore, for $i=1$ and 2 , the inverse image $U_{i}$ of the $\tau$-open set $\mathscr{P}\left(H_{i}\right)$ under $x \rightarrow V(T, x)$ is an open subset of $S(X)$, the domain of the mapping. For $x \in S(X)$, the set $V(T, x)$ is connected, being the image of the set $D(x)$, which is connected in the norm topology, under the norm continuous mapping $f \rightarrow f(T x)$. Hence, by the sentence preceding the theorem, $V(T, x) \subset G_{1} \subseteq H_{1}$ or $V(T, x) \subseteq G_{2} \subseteq H_{2}$. Thus $x \in U_{1}$ or $x \in U_{2}$, but not both. We deduce that $S(X)=$ $U_{1} \cup U_{2}$, where $U_{1} \cap U_{2}=\emptyset$. But this is impossible, since $S(X)$ is connected, and the theorem is proved.

Here we begin our second proof of the connectedness of $V(T)$. Let $P$ denote $\left\{(x, f) \in X \times X^{\prime}: x \in S(X), f \in D(x)\right\}$. We shall first prove the following general theorem:

THEOREM 2. Let $X \neq \mathbf{R}$, and let $X$ have the norm topology. Let $X^{\prime}$ have a topology satisfying

(a) $X^{\prime}$ is a topological linear space,

(b) $x \rightarrow D(x)$ is usc for all $x \in S(X)$,

(c) $D(x)$ is compact, for all $x \in S(X)$.

Then $P$ is connected as a subset of $X \times X^{\prime}$ with the product topology.

We shall then deduce:

Corollary. Let $X \neq \mathbf{R}$, and let $X \times X^{\prime}$ be topologized by the product of the norm topology and the weak* topology. Then $P$ is connected, as a subset of $X \times X^{\prime}$.

Finally we shall show that this implies Theorem 1.

Proof of Theorem 2: We shall first show that every sequence $\left\{f_{i}\right\}$ in $X^{\prime}$ which is eventually in every neighbourhood of $D(x)$ has a limit point $g$ in $D(x)$. For suppose this is false, then $D(x)$ has an open covering by sets each containing only finitely many $f_{i}$ 's. Since $D(x)$ is compact it has a finite covering by such sets. But the union of the sets in this finite covering is a neighbourhood of $D(x)$ containing only finitely many $f_{i}$ 's. Since this is a contradiction, the $f_{i}$ 's must have a limit point $g$ in $D(x)$.

Let $\pi: P \rightarrow S(X)$ denote the projection mapping $(x, f) \rightarrow x$. Then $\pi$ is a closed mapping. For suppose $K$ is a closed subset of $P$ and $x$ is a limit point of $\pi(K)$. Then there exists $\left\{\left(x_{i}, f_{i}\right)\right\} \subset K$ such that $x=\lim x_{i}=\lim \pi\left(x_{i}, f_{i}\right)$. Since $t \rightarrow D(t)$ is usc, $\left\{D\left(x_{i}\right)\right\}$ is eventually within each neighbourhood of $D(x)$. Since $f_{i} \in D\left(x_{i}\right),\left\{f_{i}\right\}$ is eventually in each neighbourhood of $D(x)$ and hence has a limit point $g$ in $D(x)$. Thus $(x, g) \in$ $\left.\overline{\left\{\left(x_{i}, f_{i}\right)\right.}\right\} \subseteq K$, whence $x \in \pi(K)$, and so $\pi(K)$ is closed. 
Suppose that $P=G_{1} \cup G_{2}$ where the $G_{i}$ 's are non-empty, disjoint, open and closed subsets of $P$. For each $x \in S(X)$ the set $\pi^{-1}(x)$ is connected because it is homeomorphic to $D(x)$. The set $\pi^{-1}(x)$ must thus be a subset of either $G_{1}$ or $G_{2}$. It follows that $\pi\left(G_{1}\right) \cap \pi\left(G_{2}\right)=\emptyset$. Since $\pi\left(G_{1}\right)$ and $\pi\left(G_{2}\right)$ are closed and cover $S(X)$, this contradicts the connectedness of $S(X)$. Hence no such $G_{i}$ 's can exist and therefore $P$ is connected.

The Corollary now follows immediately from Theorem 2, in view of Lemma 2 and the compactness of $D(x)$ in the weak* topology.

Let $X \times X^{\prime}$ have the product topology formed from the norm topology of $X$ and the weak* topology of $X^{\prime}$. If $X$ is infinite dimensional, it can be shown that the mapping $(x, f) \rightarrow f(x)$ is not continuous on $X \times X^{\prime}$, because it is unbounded on every open subset of $X \times X^{\prime}$. However:

Lemma 4. Let $F$ be a norm-bounded subset of $X^{\prime}$. Let $X \times X^{\prime}$ have the product topology formed from the norm topology of $X$ and the weak* topology of $X^{\prime}$. Then the mapping $(x, f) \rightarrow f(x)$ defined on $X \times X^{\prime}$ is a continuous mapping of the set $X \times F$ with the relative topology.

Proof: Suppose that $F$ is contained in a ball of radius $r$ centered at the origin of $X^{\prime}$. If $\left(x_{i}, f_{i}\right)$ is a net in $X \times F$,

$$
\begin{aligned}
\left|f_{i}\left(x_{i}\right)-f(x)\right| & \leqslant\left|f_{i}\left(x-x_{i}\right)\right|+\left|f_{i}(x)-f(x)\right| \\
& \leqslant r\left\|x-x_{i}\right\|+\left|f_{i}(x)-f(x)\right| .
\end{aligned}
$$

Thus $f_{i}\left(x_{i}\right)$ will converge to $f(x)$ if both $x_{i} \rightarrow x$ in the norm topology and $f_{i} \rightarrow f$ in the weak* topology, i.e. if $\left(x_{i}, f_{i}\right) \rightarrow(x, f)$ in $X \times X^{\prime}$.

Second Proof of Theorem 1. Let $X \times X^{\prime}$ be topologized as it was in the Corollary to Theorem 2 and Lemma 4 . We view the mapping $(x, f) \rightarrow[T(x)]$ defined on $P$ as the composition of the continuous functions $(x, f) \rightarrow(T(x), f) \rightarrow f[T(x)]$. (Lemma 4 shows the continuity at the final step.) Since by the Corollary to Theorem $2, P$ is connected, it follows that $V(T)$ must be connected, as it is the image of $P$ under a continuous function.

However, the numerical range $W(T)$ need not be connected:

EXAMPLE. Let $X$ be $\mathbf{R}^{2}$ or $\mathbf{C}^{2}$ with the norm given for each $x=\left(\xi_{1}, \xi_{2}\right) \in X$ by $\|x\|=$ $\max \left(\left|\xi_{1}\right|,\left|\xi_{2}\right|\right)$. Given $a=\left(\alpha_{1}, \alpha_{2}\right) \in S(X)$, and $x=\left(\xi_{1}, \xi_{2}\right) \in X$, let $f_{a}(x), T$ be defined by

$$
f_{a}(x)=\left\{\begin{array}{lll}
\bar{\alpha}_{1} \xi_{1} & \text { if } \quad\left|\alpha_{1}\right|=1 \\
\bar{\alpha}_{2} \xi_{2} & \text { if } \quad\left|\alpha_{1}\right|<1
\end{array} \quad T x=\left(\xi_{1}, 0\right)\right.
$$

Then $f_{a} \in D(a)$, and $T$ is a continuous linear operator. Also

$$
f_{a}(T a)=\left\{\begin{array}{lll}
1 & \text { if } & \left|\alpha_{1}\right|=1 \\
0 & \text { if } & \left|\alpha_{1}\right|<1
\end{array}\right.
$$


Therefore the numerical range $W(T)$ in the sense of Lumer for the semi-inner-product space corresponding to the mapping $a \rightarrow f_{a}$ is the set with exactly two elements, 1 and 0 .

\section{Upper semi-continuity and $\tau$-continuity of the mapping $x \rightarrow D(x)$ with the norm topology on $\mathrm{X}^{\prime}$.}

If $X$ has finite dimension, the norm topology coincides with the weak* topology on $X^{\prime}$, and so, by Lernma 2 , the mapping $x \rightarrow D(x)$ is upper semi-continuous and $\tau$-continuous with respect to the norm topology on $X^{\prime}$. We show that the mapping $x \rightarrow D(x)$ is also upper semi-continuous and $\tau$-continuous in this sense when $X=\left(c_{0}\right)$, but not for certain other spaces $X$ including the space $(c)$.

Let $\mathbf{F}$ denote either the real or the complex field, and $\mathbf{P}$ the set of all positive integers. We denote by $(m)$, as usual, the Banach space of all bounded mappings of $\mathbf{P}$ into $\mathbf{F}$ with the sup norm

$$
\|x\|_{\infty}=\sup \{|x(n)|: n \in \mathbf{P}\} \quad(x \in(m)),
$$

and by $(c)$ and $\left(c_{0}\right)$ the subspaces of $(m)$ consisting of all sequences that converge and converge to zero respectively. Also, as usual, we denote by $\left(l_{1}\right)$ the Banach space of all mappings $x$ of $\mathbf{P}$ into $\mathbf{F}$ such that $\|x\|_{1}=\sum_{n=1}^{\infty}|x(n)|<\infty$, normed by $\|\cdot\|_{1}$.

THEOREM 3. Let $X=\left(c_{0}\right)$. Then the mapping $x \rightarrow D(x)$ is a usc and $\tau$-continuous mapping of $S(X)$ into subsets of $X^{\prime}$ with respect to the norm topologies in $X$ and $X^{\prime}$.

Proof: To each element $f$ of $X^{\prime}$ corresponds a sequence $\left\{\lambda_{k}\right\}$ of elements of $\mathbf{F}$ such that $\sum_{k=1}^{\infty}\left|\lambda_{k}\right|=\|f\|$ and

$$
f(x)=\sum_{k=1}^{\infty} \lambda_{k} x(k) \quad(x \in X)
$$

Given $x \in S(X)$, let $E_{x}=\{k \in \mathbf{P}:|x(k)|=1\}$. Then $E_{x}$ is a non-empty finite set.

Let $a \in S(X)$ and $\varepsilon>0$. Since the set $\{k \in \mathbf{P}:|a(k)| \geqslant 1 / 2\}$ is finite

$$
\sup \left\{|a(k)|: k \in \mathbf{P} \backslash E_{a}\right\}=1-\eta
$$

with $\eta>0$. Choose $\delta$ with $0<\delta<\min (\varepsilon, \eta)$, let $b \in S(X)$ with $\|b-a\|_{\infty}<\delta$, and let $f \in D(b)$. The sequence $\left\{\lambda_{k}\right\}$ corresponding to $f$ satisfies

$$
\sum_{k=1}^{\infty}\left|\lambda_{k}\right|=\sum_{k=1}^{\infty} \lambda_{k} b(k)=1 .
$$

Therefore $\lambda_{k}=0\left(k \in \mathbf{P} \mid E_{b}\right)$ and $\lambda_{k}=\left|\lambda_{k}\right| \overline{b(k)}$ for all $k$. Let $\mu_{k}=\left|\lambda_{k}\right| \overline{a(k)}(k \in \mathbf{P})$, and let $g$ be the element of $X^{\prime}$ corresponding to the sequence $\left\{\mu_{k}\right\}$. Since $\|b-a\|_{\infty}<\eta$, we have 
$E_{b} \subseteq E_{a}$, and therefore $|a(k)|=1$ whenever $\lambda_{k} \neq 0$. Therefore

Thus $g \in D(a)$. Also

$$
\begin{gathered}
\|g\|=\sum_{k=1}^{\infty}\left|\mu_{k}\right|=\sum_{k=1}^{\infty}\left|\lambda_{k}\right|=1, \\
g(a)=\sum_{k=1}^{\infty} \mu_{k} a(k)=\sum_{k=1}^{\infty}\left|\lambda_{k}\right|=1 .
\end{gathered}
$$

$$
\begin{aligned}
\|f-g\| & =\sum_{k=1}^{\infty}\left|\lambda_{k}-\mu_{k}\right| \\
& =\sum_{k=1}^{\infty}\left|\lambda_{k}\right| \overline{b(k)}-\overline{a(k)} \mid \leqslant\|b-a\|_{\infty}<\varepsilon .
\end{aligned}
$$

Since $f$ is an arbitrary element of $D(b), g \in D(a)$, and $f=g+(f-g)$, this proves that $D(b) \subseteq D(a)+\left\{h \in X^{\prime}:\|h\|<\varepsilon\right\}$. Thus $x \rightarrow D(x)$ is usc with respect to the norm topologies in $X$ and $X^{\prime}$. Furthermore $x \rightarrow D(x)$ will be $\tau$-continuous if each $D(x)$ is compact. For each $x \in S(X), D(x)$ is a subset of $\left\{f \in S\left(X^{\prime}\right)\right.$ :support $\left.(f) \subseteq E_{x}\right\}$ which is homeomorphic to the compact set $S\left(\mathbf{F}^{k}\right)$, where $k$ is the order of $E_{x}$ and $\mathbf{F}^{k}$ has the norm $\left\|\left(t_{1}, \ldots, t_{k}\right)\right\|=\sum\left|t_{i}\right|$. Therefore since $D(x)$ is closed in the norm topology, it is compact.

That $P$ is connected when $X=\left(c_{0}\right)$ and $X^{\prime}$ has the norm topology is a special case of Theorem 2 .

THEOREM 4. Let $X$ be a linear subspace of $(m)$ such that $(c) \subseteq X$. Then the mapping $x \rightarrow D(x)$ is not upper semi-continuous with respect to the norm topologies in $X$ and $X^{\prime}$ (and therefore not $\tau$-continuous).

Proof: Given $n \in P$, let $e_{n}, a, b_{n}$ denote the elements of $X$ defined by

$$
e_{n}(k)=\left\{\begin{array}{lll}
1 & \text { if } & k=n \\
0 & \text { if } & k \neq n
\end{array}, \quad a(k)=1-\frac{1}{k}(k \in \mathbf{P}), \quad b_{n}(k)=\left\{\begin{array}{lll}
a(k) & \text { if } \quad k<n \\
1 & \text { if } \quad k \geqslant n
\end{array} .\right.\right.
$$

Let $f_{n}$ be the element of $X^{\prime}$ defined by $f_{n}(x)=x(n)(x \in X)$. Given $g \in D(a)$, we have

$$
g\left(e_{n}\right)=0(n \in \mathbf{P})
$$

for we have $\left\|a+\xi e_{n}\right\|_{\infty}=1$ whenever $|\xi| \leqslant 1 / n$, and so

$$
1 \geqslant\left|g\left(a+\xi e_{n}\right)\right|=\left|1+\xi g\left(e_{n}\right)\right| \quad(|\xi| \leqslant 1 / n),
$$

which is impossible unless $g\left(e_{n}\right)=0$. Therefore, for all $g \in D(a)$,

$$
\left\|f_{n}-g\right\| \geqslant\left|\left(f_{n}-g\right)\left(e_{n}\right)\right|=1 \quad(n \in \mathbf{P}) .
$$

However $f_{n} \in D\left(b_{n}\right)$ and $\left\|b_{n}-a\right\|_{\infty}=1 / n$. Thus $b_{n}$ tends to $a$, but $D\left(b_{n}\right) \nsubseteq D(a)+U_{1}$, where $U_{1}=\left\{f \in X^{\prime}:\|f\|<1\right\}$, and the result follows.

THEOREM 5. Let $X=\left(l_{1}\right)$. Then the mapping $x \rightarrow D(x)$ is not upper semi-continuous with respect to the norm topologies in $X$ and $X^{\prime}$ (and therefore not $\tau$-continuous). 
Proof: Given $n \in \mathbf{P}$, let $a, b_{n}$ be the elements of $S(X)$ defined by

Then

$$
a(k)=1 / 2^{k} \quad(k \in \mathbf{P}), \quad b_{n}(k)= \begin{cases}2^{n} /\left(2^{n}-1\right) 2^{k} & (k \leqslant n) \\ 0 & (k>n) .\end{cases}
$$

$$
\left\|a-b_{n}\right\|_{1}=\frac{1}{2^{n}-1} \sum_{k=1}^{n} \frac{1}{2^{k}}+\sum_{k=n+1}^{\infty} \frac{1}{2^{k}}=\frac{1}{2^{n-1}} .
$$

Let $f_{n}$ be the functional defined by

$$
f_{n}(x)=\sum_{k=1}^{n} x(k) \quad(x \in X) .
$$

and let $g \in D(a)$. Since $a(k)>0(k \in \mathbf{P})$, we have

$$
g(x)=\sum_{k=1}^{\infty} x(k) \quad(x \in X) .
$$

Also $f_{n} \in D\left(b_{n}\right)$ and $\left\|f_{n}-g\right\|=1(n \in \mathbf{P})$. Thus the mapping $x \rightarrow D(x)$ is not upper semicontinuous with respect to the norm topologies in $X$ and $X^{\prime}$.

Note added in proof: Our lemma 2 is known, cf. Theorem 4.3, D. F. Cudia: The Geometry of Banach spaces. Smoothness. Trans. Amer. Math. Soc. I10, 284-314 (1964).

\section{REFERENCES}

[1] Bauter, F. L., On the Field of Values Subordinate to a Norm, Numer. Math. 4, 103-111 (1962).

[2] Berge, C., Topological Spaces (The MacMillan Company, New York 1963).

[3] Kelley; J. L., NamoKa, I., and co-authors, Linear Topological Spaces (D. van Nostrand Company, New York 1963).

[4] Lumer, G., Semi-Inner-Product Spaces, Trans. Amer. Math. Soc. 100, $29-43$ (1961).

[5] Nirschl, N. and Schnelder, H., The Bauer Fields of Values of a Matrix, Numer. Math. 6, 355-365 (1964).

[6] RiCKART, C. E., General Theory of Banach Algebras (D. van Nostrand Company, New York 1960).

[7] STONE, M. H., Linear Transformations in Hilbert Space and Their Applications to Analysis (Amer. Math. Soc., New York 1932 [Col. Publ., Nr. 15]).

University of Edinburgh University of Wisconsin 